\title{
Frequent blood donors risk iron deficiency
}

$\mathrm{D}$ r. Mindy Goldman of the Canadian Blood Services had just finished a talk on blood donors' safety at the University of Toronto when a few doctors and nurses approached her. "You know, I used to donate every 56 days, then my hemoglobin crashed," one said. Another said she too became iron-deficient after donating regularly. "These are not rare situations," says Goldman, the blood services' medical director of donor and clinical services.

Dr. Bruce Newman, Southeastern Michigan Region medical director for the American Red Cross, is more blunt: "We are actually making people iron deficient." He points out that blooddonation-induced iron deficiency can cause people to be "a little more tired or just not able to endure as well" even before it leads to decreased hemoglobin and anemia.

Blood centres test donors' hemoglobin, but do not measure their iron levels. In 2011, a multisite study, REDS II (Retrovirus Epidemiology Donor Study-II), funded by the National Institutes of Health, documented iron deficiency and iron depletion in blood donors. It found a high prevalence of these conditions in so-called repeat donors: women who give blood twice or men who donate three times annually. In August 2013, Goldman published a Canadian study in Transfusion with similar findings. That study tested ferritin in 550 Ottawa blood donors and found about two-thirds of female and one-third of male repeat donors had low or absent iron stores.

The conundrum for Goldman and her colleagues is that Canadian Blood Services relies on loyal, repeat donors for nearly $90 \%$ of Canada's blood donations. "We have to take action in a responsible way for both our donors and our patients," Goldman says.

Canadian Blood Services will continue to encourage men to donate about seven times per year or once every 56 days, a frequency matched in the United States. Europe limits donations to six

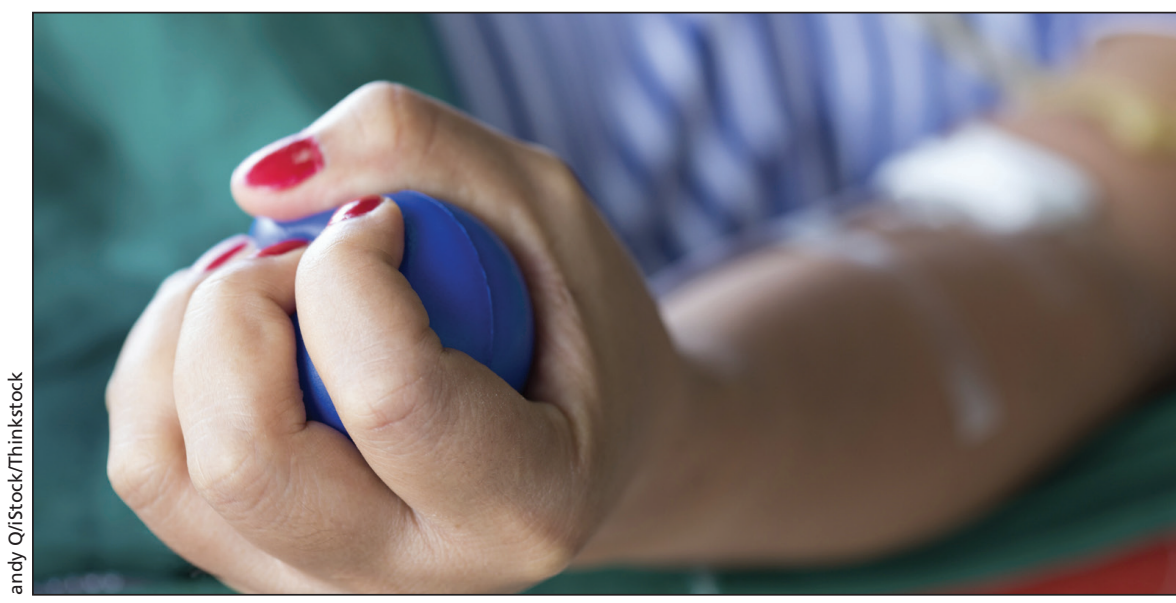

Canadian Blood Services will soon ask women to donate four times annually instead of six.

times annually for men, four for women. The United Kingdom and Hong Kong limit men to four, women to three.

Though Canadian Blood Services will contact female donors less frequently, there are no plans to decrease the number of times male or female Canadians are permitted to donate, which would require government approval. Health Canada spokesperson Leslie Meerburg wrote in an email that the agency "is aware of the issue of iron stores and frequent donation," but Goldman said that Blood Services has "not heard anything from Health Canada on the donor iron issue."

The risk of frequent donations was brought home to Newman in a dramatic way after his wife gave blood five times one year. Her doctor diagnosed anemia and referred her for a colonoscopy to search for a source of blood loss. Though she mentioned she was a blood donor, when the results of the colonoscopy were normal, the doctor advised her to undergo an upper gastrointestinal (GI) endoscopy. "Most doctors are not aware of the relationship between blood donations and anemia," says Newman, "so they don't think about it."

Blood donors in Canada report similar experiences, says Goldman. "Some individuals may have quite an extensive GI investigation for blood loss, when maybe the first step could have been to ask them, 'Are you donating every 56 days?"' For frequent donors who have low hemoglobin or ferritin levels, Newman says the best medical advice is to start taking iron supplements and temporarily stop donating blood.

The American Red Cross now recommends iron supplements for women who donate two or more times and for men who donate three or more times in a twelve-month period. And a transfusion industry group, Advancing Transfusion and Cellular Therapies Worldwide, advises all blood collecting organizations to protect donors against iron deficiency by methods such as limiting the number of donations, recommending donors take iron supplements or directly providing donors with replacement iron.

Later this year, Canadian Blood Services will begin taking an extra tube of blood from about 10000 donors as part of a study to test the effectiveness of measuring ferritin at the time of blood donation. If the level is low, Canadian Blood Services will write the donor a letter encouraging him or her to start iron supplements and to see a physician.

Goldman expects about $30 \%$ of the donors tested will have low or absent iron. She hopes each donor will raise awareness around blood-donationinduced iron deficiency. - Miriam Shuchman MD, Toronto, Ont.

CMAJ 2014. DOI:10.1503/cmaj.109-4807 